\title{
A NOTE ON CONTROLLABILITY OF SEMILINEAR INTEGRODIFFERENTIAL SYSTEMS IN BANACH SPACES
}

\author{
K. BALACHANDRAN and R. SAKTHIVEL \\ Bharathiar University \\ Department of Mathematics \\ Coimbatore 641046 , India
}

(Received March, 1998; Revised January, 1999)

\begin{abstract}
Sufficient conditions for controllability of semilinear integrodifferential systems in a Banach space are established. The results are obtained by using the Schaefer fixed-point theorem.
\end{abstract}

Key words: Controllability, Semilinear Integrodifferential System, Fixed-Point Theorem.

AMS subject classifications: 93B05.

\section{Introduction}

Controllability of linear and nonlinear systems represented by ordinary differential equations in finite-dimensional space has been extensively studied. Several authors have extended the concept to infinite-dimensional systems in Banach spaces with bounded operators. Naito [8,9] studied the controllability of semilinear systems whereas Yamamoto and Park [13] considered the same problem for parabolic equation with uniformly bounded nonlinear term. Lasiecka and Triggiani [5] studied exact controllability of abstract semilinear equations. Chukwu and Lenhart [3] discussed the controllability of nonlinear systems in abstract spaces and Naito [10] established the controllability for nonlinear Volterra integrodifferential systems. Do [4] and Zhou [14] investigated the approximate controllability for a class of semilinear abstract equations. Recently Balachandran et al. [1,2] established sufficient conditions for the controllability of nonlinear integrodifferential systems in Banach spaces by using Schauder's fixed-point theorem. The purpose of this paper is to study the controllability of semilinear integrodifferential systems in Banach spaces by suitably applying the Schaefer fixed-point theorem.

\section{Preliminaries}

Consider the semilinear integrodifferential system

$\dot{x}(t)=A\left[x(t)+\int_{0}^{t} F(t-s) x(s) d s\right]+(B u)(t)+f\left(t, x(t), \int_{0}^{t} g(t, s, x(s)) d s\right), t \in J=[0, b]$, 


$$
x(0)=x_{0}
$$

where the state $x(\cdot)$ takes values in a Banach space $X$ and the control function $u(\cdot)$ is given in $L^{2}(J, U)$, a Banach space of admissible control functions with $U$ as a Banach space. Here $A$ is the generator of a strongly continuous semigroup, $B$ is a bounded linear operator from $U$ into $X$, and $g: J \times J \times X \rightarrow X$ and $f: J \times X \times X \rightarrow X$ are given functions. $F(t): Y \rightarrow Y$ and for $x(\cdot)$ continuous in $Y, A F(\cdot) x(\cdot) \in$ $L^{1}([0, b], X) . \quad F(t) \in B(X), t \in J$ and for some $x \in X, F^{\prime}(t) x$ is continuous in $t \in$ $[0, b]$, where $B(X)$ is the space of all bounded linear operators on $X$, and $Y$ is the Banach space formed from $D(A)$, the domain of $A$ endowed with the graph norm.

We need the following fixed point theorem due to Schaefer [12].

Schaefer Theorem: Let $S$ be a convex subset of a normed linear space $E$ and $0 \in S$. Let $F: S \rightarrow S$ be a completely continuous operator and let

$$
\zeta(F)=\{x \in S ; x=\lambda F x \text { for some } 0<\lambda<1\} \text {. }
$$

Then either $\zeta(F)$ is unbounded or $F$ has a fixed point.

The system (1) has a mild solution of the following form [11]:

$$
x(t)=R(t) x_{0}+\int_{0}^{t} R(t-s)\left[(B u)(s)+f\left(s, x(s), \int_{0}^{s} g(s, \tau, x(\tau)) d \tau\right)\right] d s
$$

where $R(t)$ is a resolvent operator [6].

In order to study the controllability problem of (1), we consider the following system as in [7]:

$$
\begin{gathered}
\dot{x}(t)=\lambda A\left[x(t)+\int_{0}^{t} F(t-s) x(s) d s\right]+\lambda(B u)(t) \\
\left.+\lambda f\left(t, x(t), \int_{0}^{t} g(t, s x(s)) d s\right)\right), \lambda \in(0,1), t \in J, \\
x(0)=x_{0} .
\end{gathered}
$$

Then for system (3), there exists a mild solution of the form

$$
x(t)=\lambda R(t) x_{0}+\lambda \int_{0}^{t} R(t-s)\left[(B u)(s)+f\left(s, x(s), \int_{0}^{s} g(s, \tau, x(\tau)) d \tau\right)\right] d s .
$$

Definition: System (1) is said to be controllable on the interval $J$ if for every $x_{0}, x_{1} \in X$, there exists a control $u \in L^{2}(J, U)$ such that the solution $x(\cdot)$ of (1) satisfies $x(b)=x_{1}$.

We assume the following hypotheses:

(i) The resolvent operator $R(t)$ is compact such that

$$
\max _{t>0}\|R(t)\| \leq M_{1}
$$


where $M_{1}>0$.

(ii) The linear operator $W$ from $L^{2}(J, U)$ into $X$, defined by

$$
W u=\int_{0}^{b} R(b-s) B u(s) d s
$$

has an invertible operator $W^{-1}$ defined on $L^{2}(J, U) / k e r W$ and there exist positive constants $M_{2}, M_{3}$ such that $\|B\| \leq M_{2}$ and $\left\|W^{-1}\right\| \leq M_{3}$.

(iii) For each $t \in J$, the function $g(t, s, \cdot): X \rightarrow X$ is continuous and for each $x \in X$, the function $g(\cdot, \cdot, x): J \times J \rightarrow X$ is strongly measurable.

(iv) For each $t \in J$, the function $f(t, \cdot, \cdot): X \times X \rightarrow X$ is continuous and for each $x, y \in X$, the function $f(\cdot, x, y): J \rightarrow X$ is strongly measurable.

(v) For every positive integer $k$, there exists $h_{k} \in L^{1}(0, b)$ such that for a.a. $t \in J$

$$
\sup _{\|x\| \leq k \|}\left\|f\left(t, x(t), \int_{0}^{t} g(t, s, x(s)) d s\right)\right\| \leq h_{k}(t) .
$$

(vi) There exists a continuous function $m: J \times J \rightarrow[0, \infty)$ such that

$$
\|g(t, s, x)\| \leq m(t, s) \Omega(\|x\|), \quad t, s \in J, \quad x \in X,
$$

where $\Omega:[0, \infty) \rightarrow(0, \infty)$ is a continuous nondecreasing function.

(vii) There exists a continuous function $p: J \rightarrow[0, \infty)$ such that

$$
\|f(t, x, y)\| \leq p(t) \Omega_{0}(\|x\|+\|y\|), t \in J \quad x, y \in X
$$

where $\Omega_{0}:[0, \infty) \rightarrow(0, \infty)$ is a continuous nondecreasing function.

(viii)

$$
\int_{0}^{b} \widehat{m}(s) d s<\int_{c}^{\infty} \frac{d s}{\Omega_{0}(s)+\Omega(s)}
$$

where $c=M_{1}\left(\left\|x_{0}\right\|\right)+M_{1} N b, \widehat{m}(t)=\max \left\{M_{1} p(t), L m(t, t)\right\}$,

$$
\begin{gathered}
N=M_{2} M_{3}\left(\left\|x_{1}\right\|+M_{1}\left\|x_{0}\right\|+M_{1} \int_{0}^{b} p(s) \Omega_{0}(\|x\|\right. \\
\left.\left.+L \int_{0}^{s} m(s, \tau) \Omega(\|x\|) d \tau\right) d s\right)
\end{gathered}
$$

\section{Main Result}

Theorem: If the hypotheses (i)-(viii) are satisfied, then system (1) is controllable on $J$.

Proof: Using hypothesis $(i i)$ for an arbitrary function $x(\cdot)$, define the control 


$$
u(t)=W^{-1}\left[x_{1}-R(b) x_{0}-\int_{0}^{b} R(b-s) f\left(s, x(s), \int_{0}^{s} g(s, \tau, x(\tau)) d \tau\right) d s\right](t) .
$$

We shall now show that when using this control the operator defined by

$$
\begin{aligned}
& (F x)(t)=R(t) x_{0}+\int_{0}^{t} R(t-s)[(B u)(s) \\
& \left.+f\left(s, x(s), \int_{0}^{s} g(s, \tau, x(\tau)) d \tau\right)\right] d s, t \in J
\end{aligned}
$$

has a fixed point. This fixed point is then a solution of equation (2).

Clearly, $(F x)(b)=x_{1}$, which means that the control $u$ steers the semilinear integrodifferential system from the initial state $x_{0}$ to $x_{1}$ in time $b$, provided we can obtain a fixed point of the nonlinear operator $F$.

First, we obtain a priori bounds for the following equation:

$$
\begin{aligned}
& x(t)=\lambda R(t) x_{0}+\lambda \int_{0}^{t} R(t-\eta) B W^{-1}\left[x_{1}-R(b) x_{0}\right. \\
& \left.-\int_{0}^{b} R(b-s) f\left(s, x(s), \int_{0}^{s} g(s, \tau, x(\tau)) d \tau\right) d s\right](\eta) d \eta \\
& +\lambda \int_{0}^{t} R(t-s) f\left(s, x(s), \int_{0}^{s} g(s, \tau, x(\tau)) d \tau\right) d s .
\end{aligned}
$$

We have, from the assumptions,

$$
\begin{gathered}
\|x(t)\| \leq M_{1}\left\|x_{0}\right\|+\int_{0}^{t}\|R(t-\eta)\| M_{2} M_{3}\left[\left\|x_{1}\right\|+M_{1}\left\|x_{0}\right\|\right. \\
\left.+M_{1} \int_{0}^{b} p(s) \Omega_{0}\left(\|x(s)\|+\int_{0}^{s} m(s, \tau) \Omega(\|x(\tau)\|) d \tau\right) d s\right] d \eta \\
+M_{1} \int_{0}^{t} p(s) \Omega_{0}\left(\|x(s)\|+\int_{0}^{s} m(s, \tau) \Omega(\|x(\tau)\|) d \tau\right) d s \\
\leq M_{1}\left\|x_{0}\right\|+\int_{0}^{t} M_{1} N d s
\end{gathered}
$$




$$
\begin{gathered}
+M_{1} \int_{0}^{t} p(s) \Omega_{0}\left(\|x(s)\|+\int_{0}^{s} m(s, \tau) \Omega(\|x(\tau)\|) d \tau\right) d s \\
\leq M_{1}\left\|x_{0}\right\|+M_{1} N b+M_{1} \int_{0}^{t} p(s) \Omega_{0}(\|x(s)\| \\
\left.+\int_{0}^{s} m(s, \tau) \Omega(\|x(\tau)\|) d \tau\right) d s .
\end{gathered}
$$

Denoting by $v(t)$ the right-hand side of the above inequality, we have $v(0)=$ $M_{1}\left\|x_{0}\right\|+M_{1} N b,\|x(t)\| \leq v(t)$ and

$$
\begin{aligned}
v^{\prime}(t)= & M_{1} p(t) \Omega_{0}\left(\|x(t)\|+\int_{0}^{t} m(t, \tau) \Omega(\|x(\tau)\|) d \tau\right) \\
& \leq M_{1} p(t) \Omega_{0}\left(v(t)+\int_{0}^{t} m(t, \tau) \Omega(v(\tau)) d \tau\right) .
\end{aligned}
$$

Let

$$
w(t)=v(t)+\int_{0}^{t} m(t, \tau) \Omega(v(\tau)) d \tau
$$

Then $w(0)=v(0)=c, v(t) \leq w(t)$, and

$$
\begin{gathered}
w^{\prime}(t)=v^{\prime}(t)+m(t, t) \Omega(v(t)) \leq M_{1} p(t) \Omega_{0}(w(t))+m(t, t) \Omega(w(t)) \\
\leq \widehat{m}(t)\left[\Omega_{0}(w(t))+\Omega(w(t))\right] .
\end{gathered}
$$

This implies that

$$
\int_{w(0)}^{w(t)} \frac{d s}{\Omega_{0}(s)+\Omega(s)} \leq \int_{0}^{b} \widehat{m}(s) d s<\int_{c}^{\infty} \frac{d s}{\Omega_{0}(s)+\Omega(s)}, \quad t \in J,
$$

which in turn implies that there is a constant $K$ such that $w(t) \leq K, t \in J$, and hence $\|x(t)\| \leq K, t \in J$, where $K$ depends only on $b$ and on the functions $m, \Omega_{0}$, and $\Omega$.

Second, we must prove that the operator $F: C=C(J, X) \rightarrow C$ defined by

$$
\begin{aligned}
& (F x)(t)=R(t) x_{0}+\int_{0}^{t} R(t-\eta) B W^{-1}\left[x_{1}-R(b) x_{0}\right. \\
& \left.-\int_{0}^{b} R(b-s) f\left(s, x(s), \int_{0}^{s} g(s, \tau, x(\tau)) d \tau\right) d s\right](\eta) d \eta
\end{aligned}
$$




$$
+\int_{0}^{t} R(t-s) f\left(s, x(s), \int_{0}^{s} g(s, \tau, x(\tau)) d \tau\right) d s
$$

is a completely continuous operator.

Let $B_{k}=\{x \in C:\|x\| \leq k\}$ for some $k \geq 1$. We first show that $F$ maps $B_{k}$ into an equicontinuous family. Let $x \in B_{k}$ and $t_{1}, t_{2} \in J$. Then if $0<t_{1}<t_{2} \leq b$,

$$
\begin{aligned}
& \left\|(F x)\left(t_{1}\right)-(F x)\left(t_{2}\right)\right\| \leq\left\|R\left(t_{1}\right)-R\left(t_{2}\right)\right\|\left\|x_{0}\right\| \\
& +\| \int_{0}^{t_{1}}\left[R\left(t_{1}-\eta\right)-R\left(t_{2}-\eta\right)\right] B W^{-1}\left[x_{1}-R(b) x_{0}\right. \\
& \left.-\int_{0}^{b} R(b-s) f\left(s, x(s), \int_{0}^{s} g(s, \tau, x(\tau)) d \tau\right) d s\right](\eta) d \eta \| \\
& +\| \int_{t_{1}}^{t_{2}} R\left(t_{2}-\eta\right) B W^{-1}\left[x_{1}-R(b) x_{0}\right. \\
& \left.-\int_{0}^{b} R(b-s) f\left(s, x(s), \int_{0}^{s} g(s, \tau, x(\tau)) d \tau\right) d s\right](\eta) d \eta \| \\
& +\left\|\int_{0}^{t_{1}}\left[R\left(t_{1}-s\right)-R\left(t_{2}-s\right)\right] f\left(s, x(s), \int_{0}^{s} g(s, \tau, x(\tau)) d \tau\right) d s\right\| \\
& +\left\|\int_{t_{1}}^{t_{2}} R\left(t_{2}-s\right) f\left(s, x(s), \int_{0}^{s} g(s, \tau, x(\tau)) d \tau\right) d s\right\| \\
& \leq\left\|R\left(t_{1}\right)-R\left(t_{2}\right)\right\|\left\|x_{0}\right\| \\
& +\int_{0}^{t_{1}}\left\|R\left(t_{1}-\eta\right)-R\left(t_{2}-\eta\right)\right\| M_{2} M_{3}\left[\left\|x_{1}\right\|+M_{1} e^{\omega b}\left\|x_{0}\right\|\right. \\
& \left.+M_{1} \int_{0}^{b} e^{\omega(b-s)} h_{k}(s) d s\right] d \eta \\
& +\int_{t_{1}}^{t_{2}}\left\|R\left(t_{2}-\eta\right)\right\| M_{2} M_{3}\left[\left\|x_{1}\right\|+M_{1} e^{\omega b}\left\|x_{0}\right\|\right. \\
& \left.+M_{1} \int_{0}^{b} e^{\omega(b-s)} h_{k}(s) d s\right] d \eta
\end{aligned}
$$




$$
\begin{gathered}
+\int_{0}^{t_{1}}\left\|\left[R\left(t_{1}-s\right)-R\left(t_{2}-s\right)\right]\right\| h_{k}(s) d s \\
+\int_{t_{1}}^{t_{2}}\left\|R\left(t_{2}-s\right)\right\| h_{k}(s) d s
\end{gathered}
$$

The right-hand side tends to zero as $t_{2}-t_{1} \rightarrow 0$, since the compactness of $R(t)$ for $t>0$ implies the continuity in the uniform operator topology.

Thus, $F$ maps $B_{k}$ into an equicontinuous family of functions. It is easy to see that the family $F B_{k}$ is uniformly bounded.

Next, we show that $\overline{F B_{k}}$ is compact. Since we have shown $F B_{k}$ is an equicontinuous collection, it suffices by the Arzela-Ascoli theorem, to show that $F$ maps $B_{k}$ into a precompact set in $X$. fine

Let $0<t \leq b$ be fixed and $\epsilon$ a real number satisfying $0<\epsilon<t$. For $x \in B_{k}$, we de-

$$
\begin{gathered}
\left(F_{\epsilon} x\right)(t)=R(t) x_{0}+\int_{0}^{t-\epsilon} R(t-\eta) B W^{-1}\left[x_{1}-R(b) x_{0}\right. \\
\left.-\int_{0}^{b} R(b-s) f\left(s, x(s), \int_{0}^{s} g(s, \tau, x(\tau)) d \tau\right) d s\right](\eta) d \eta \\
+\int_{0}^{t-\epsilon} R(t-s) f\left(s, x(s), \int_{0}^{s} g(s, \tau, x(\tau)) d \tau\right) d s \\
=R(t) x_{0}+R(\epsilon) \int_{0}^{t-\epsilon} R-1(\epsilon) R(t-\eta) B W-1\left[x_{1}-R(b) x_{0}\right. \\
\left.-\int_{0}^{b} R(b-s) f\left(s, x(s), \int_{0}^{s} g(s, \tau, x(\tau)) d \tau\right) d s\right](\eta) d \eta \\
+R(\epsilon) \int_{0}^{t-\epsilon} R-1(\epsilon) R(t-s) f\left(s, x(s), \int_{0}^{s} g(s, \tau, x(\tau)) d \tau\right) d s .
\end{gathered}
$$

Since $R(t)$ is a compact operator, the set $Y_{\epsilon}(t)=\left\{\left(F_{\epsilon} x\right)(t): x \in B_{k}\right\}$ is precompact in $X$ for every $\epsilon, 0<\epsilon<t$. Moreover, for every $x \in B_{k}$, we have

$$
\left\|(F x)(t)-\left(F_{\epsilon} x\right)(t)\right\| \leq \int_{t-\epsilon}^{t} \| R(t-\eta) B W^{-1}\left[x_{1}-R(b) x_{0}\right.
$$




$$
\begin{gathered}
\left.-\int_{0}^{b} R(b-s) f\left(s, x(s), \int_{0}^{s} g(s, \tau, x(\tau)) d \tau\right) d s\right](\eta) \| d \eta \\
+\int_{t-\epsilon}^{t}\left\|R(t-s) f\left(s, x(s), \int_{0}^{s} g(s, \tau, x(\tau)) d \tau\right)\right\| d s \\
\leq \int_{t-\epsilon}^{t}\|R(t-\eta)\| M_{2} M_{3}\left[\left\|x_{1}\right\|+M_{1} e^{\omega b}\left\|x_{0}\right\|\right. \\
\left.+M_{1} \int_{0}^{b} e^{\omega(b-s)} h_{k}(s) d s\right] d \eta \\
+\int_{t-\epsilon}^{t}\|R(t-s)\| h_{k}(s) d s .
\end{gathered}
$$

Therefore, there are precompact sets arbitrarily close to the set $\left\{(F x)(t): x \in B_{k}\right\}$. Hence the set $\left\{(F x)(t): x \in B_{k}\right\}$ is precompact in $X$.

It remains to show that $F: C \rightarrow C$ is continuous. Let $\left\{x_{n}\right\}_{0}^{\infty} \subseteq C$ with $x_{n} \rightarrow x$ in $C$. Then there is an integer $r$ such that $\left\|x_{n}(t)\right\| \leq r$ for all $n$ and $t \in J$, so $x_{n} \in B_{r}$ and $x \in B_{r}$.

By $(i v)$,

$$
f\left(t, x_{n}(t), \int_{0}^{t} g\left(t, s, x_{n}(s)\right) d s\right) \rightarrow f\left(t, x(t), \int_{0}^{t} g(t, s, x(s)) d s\right)
$$

for each $t \in J$ and since

$$
\left\|f\left(t, x_{n}(t), \int_{0}^{t} g\left(t, s, x_{n}(s)\right) d s\right)-f\left(t, x(t), \int_{0}^{t} g(t, s, x(s)) d s\right)\right\| \leq 2 h_{r}(t)
$$

we have by dominated convergence theorem,

$$
\begin{gathered}
\left\|F x_{n}-F x\right\|=\sup _{t \in J} \| \int_{0}^{t} R(t-\eta) B W^{-1}\left[\int_{0}^{b} R(b-s)\right. \\
\left.\left[f\left(s, x_{n}(s), \int_{0}^{s} g\left(s, \tau, x_{n}(\tau)\right) d \tau\right)-f\left(s, x(s), \int_{0}^{s} g(s, \tau, x(\tau)) d \tau\right)\right] d s\right](\eta) d \eta \\
+\int_{0}^{t} R(t-s)\left[f\left(s, x_{n}(s), \int_{0}^{s} g\left(s, \tau, x_{n}(\tau)\right) d \tau\right)\right.
\end{gathered}
$$




$$
\begin{gathered}
\left.-f\left(s, x(s), \int_{0}^{s} g(s, \tau, x(\tau)) d \tau\right)\right] d s \| \\
\leq \int_{0}^{b}\|R(t-\eta)\| M_{2} M_{3}\left[M_{1} \int_{0}^{b} e^{\omega(b-s)} \| f\left(s, x_{n}(s), \int_{0}^{s} g\left(s, \tau, x_{n}(\tau)\right) d \tau\right)\right. \\
\left.-f\left(s, x(s), \int_{0}^{s} g(s, \tau, x(\tau)) d \tau\right) \| d s\right] d \eta \\
+\int_{0}^{b}\|R(t-s)\| \| f\left(s, x_{n}(s), \int_{0}^{s} g\left(s, \tau, x_{n}(\tau)\right) d \tau\right) \\
-f\left(s, x(s), \int_{0}^{s} g(s, \tau, x(\tau)) d \tau\right) \| d s \rightarrow 0, \text { as } n \rightarrow \infty .
\end{gathered}
$$

Thus $F$ is continuous. This completes the proof that $F$ is completely continuous.

Finally, the set $\zeta(F)=\{x \in C: x=\lambda F x, \lambda \in(0,1)\}$ is bounded, as we proved in the first step. Consequently, by Schaefer's theorem, the operator $F$ has a fixed point in $C$. This means that any fixed point of $F$ is a mild solution of (1) on $J$ satisfying $(F x)(t)=x(t)$. Thus system (1) is controllable on $J$.

\section{Acknowledgement}

This work is supported by CSIR-New Delhi, India (Grant No. 25(89) 97EMR-II).

\section{References}

[1] Balachandran, K., Dauer, J.P. and Balasubramaniam, P., Controllability of nonlinear integrodifferential systems in Banach spaces, J. of Optim. Theory and Appl. 84 (1995), 83-91.

[2] Balachandran, K., Dauer, J.P. and Balasubramaniam, P., Controllability of semilinear integrodifferential systems in Banach spaces, J. of Math. Sys., Estim. and Control 6 (1996), 477-480.

[3] Chukwu, E.N. and Lenhart, S.M., Controllability questions for nonlinear systems in abstract spaces, J. of Optim. Theory and Appl. 68 (1991), 437-462.

[4] Do, V.N., A note on approximate controllability of semilinear systems, Syst. and Control Letters 12 (1989), 365-371.

[5] Lasiecka, I. and Triggiani, R., Exact controllability of semilinear abstract systems with application to waves and plates boundary control problems, Appl. of Math. Optim. 23 (1991), 109-154.

[6] Lin, Y. and Liu, J.H., Semilinear integrodifferential equations with nonlocal Cauchy problem, Nonl. Anal. 26 (1996), 1023-1033. 
[7] Ntouyas, S.K. and Tsamatos, P.Ch., Global existence for semilinear evolution equations with nonlocal conditions, J. of Math. Anal. and Appl. 210 (1997), 679-687.

[8] Naito, K., Controllability of semilinear control systems dominated by the linear part, SIAM J. on Contr. and Optim. 25 (1987), 715-722.

[9] Naito, K., Approximate controllability for trajectories of semilinear control systems, J. of Optim. Theory and Appl. 60 (1989), 57-65.

[10] Naito, K., On controllability for a nonlinear Volterra equation, Nonl. Anal.: Theory, Methods and Appl. 18 (1992), 99-108.

[11] Pazy, A., Semigroups of Linear Operators and Applications to Partial Differential Equations, Springer Verlag, New York 1983.

[12] Schaefer, H., Uber die methode der a priori schranken, Math. Annalem 129 (1955), 415-416.

[13] Yamamoto, M. and Park, J.Y., Controllability for parabolic equations with uniformly bounded nonlinear terms, J. of Optim. Theory and Appl. 66 (1990), 515532.

[14] Zhou, H.X., Approximate controllability for a class of semilinear abstract equations, SIAM J. on Control and Optim. 21 (1983), 551-565. 


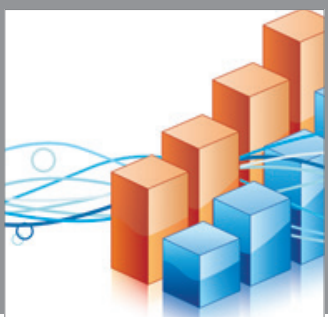

Advances in

Operations Research

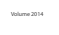

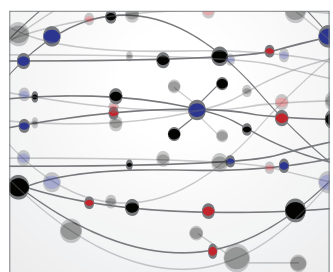

\section{The Scientific} World Journal
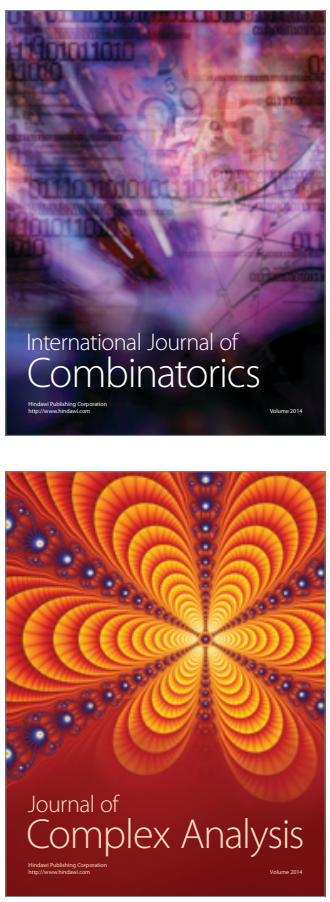

International Journal of

Mathematics and

Mathematical

Sciences
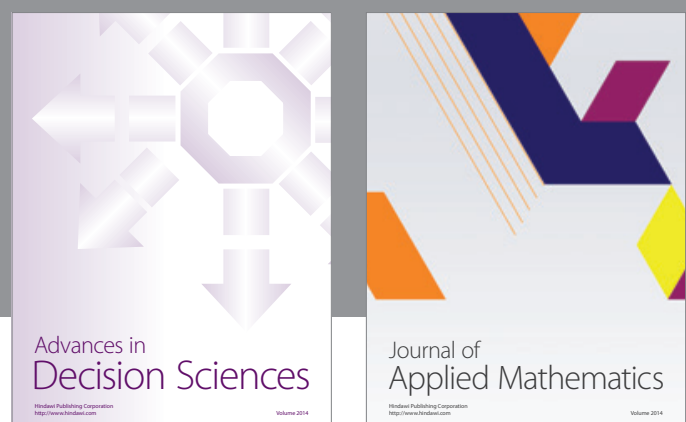

Journal of

Applied Mathematics
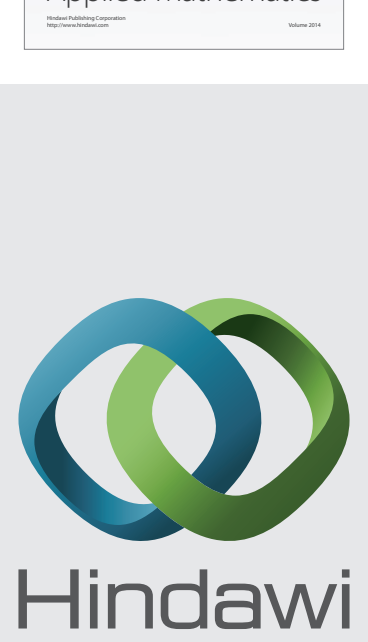

Submit your manuscripts at http://www.hindawi.com
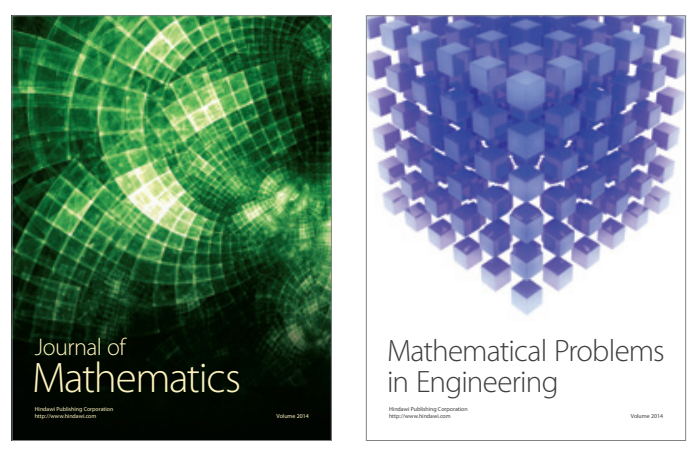

Mathematical Problems in Engineering
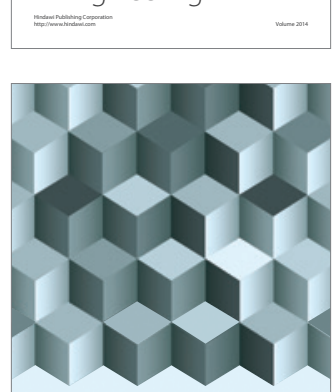

Journal of

Function Spaces
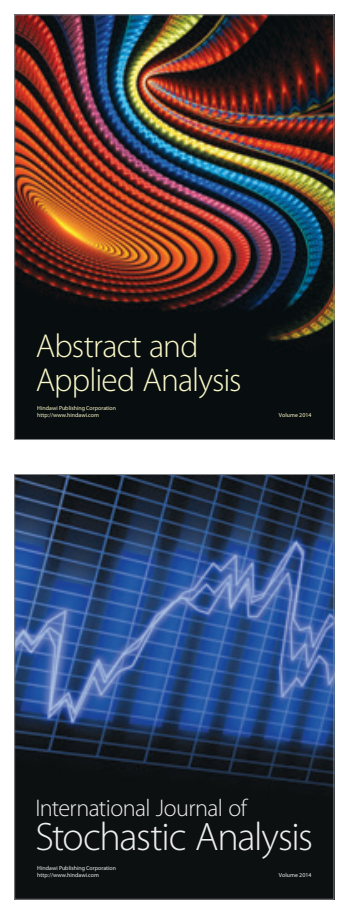

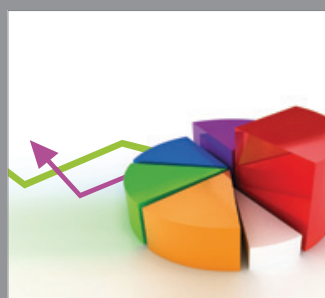

ournal of

Probability and Statistics

Promensencen
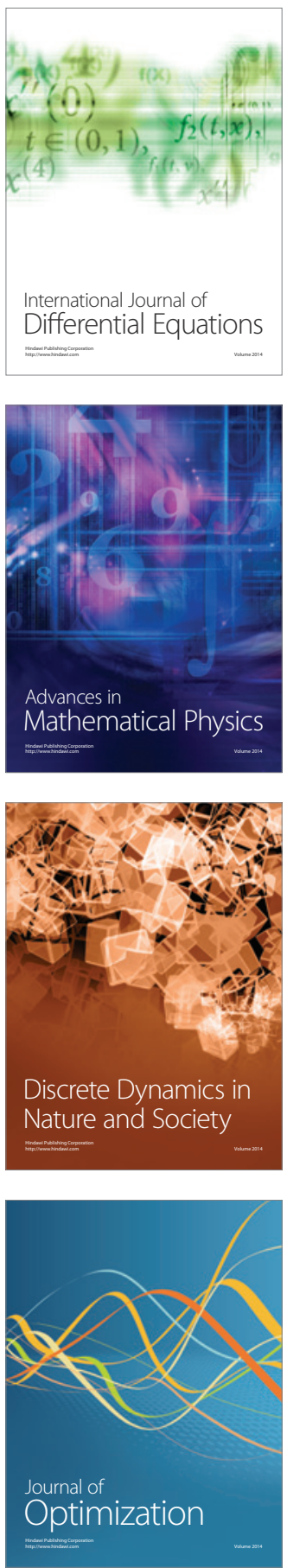